Apidologie, 1976, 7 (3), 243-248

\title{
NACHWEIS VON HETEROSIS FÜR DAS MERKMAL TEMPERATURREGULIERUNG BEI DER HONIGBIENE (APIS MELLIFICA)
}

Démonstration du phénomène d'hétérosis pour le caractère régulation thermique chez l'abeille (Apis mellifica)

Dorothea BRÜCKNER

Institut für Bienenkunde 637 Oberursel/Ts., Im Rothkopf 5, und Zoologisches Institut der Universität 8 München 2, Seidlstr 25 B.R.D., R.F.A.

\section{SUMMARY}

EVIDENCE OF HETEROSIS FOR THE TRAIT THERMOREGULATION IN THE HONEY BEE (Apis mellifica)

With experiments in which the thermoregulation of groups of 50 workers from two strongly inbred lines of Apis mellifica carnica and their hybrids was measured it could be shown that the trait thermoregulation exhibits heterosis.

\section{ZUSAMMENFASSUNG}

Bei Messungen an Gruppen von je 50 Arbeiterinnen aus zwei stark ingezüchteten, nicht verwandten Linien von Apis mellifica carnica und deren Hybriden konnte nachgewiesen werden, dass Heterosis beim Merkmal Temperaturregulierung auftritt.

\section{EINLEITUNG}

In vorangegangenen Untersuchungen zur Temperaturregulierung von ingezüchteten und nicht-ingezüchteten Völkern der Honigbiene konnte gezeigt werden, daß die Inzuchtvölker den Nicht-Inzuchtvölkern in ihrer 
Fähigkeit, die Temperatur zu regulieren, unterlegen sind (BRüCKNER, 1975) : Die Inzuchtvölker erreichten bei niedrigen Umgebungstemperaturen weniger hohe und weniger konstante Brutnesttemperaturen als die Nicht-Inzuchtvölker. Es konnte gezeigt werden, daß die Brutnesttemperatur der Inzuchtvölker zudem stark von der Umgebungstemperatur abhängig ist; bei der niedrigsten Umgebungstemperatur trat auch die niedrigste Brutnest temperatur auf. Auch bei Messungen an Gruppen von je 50 Arbeiterinnen bei verschiedenen niedrigen Temperaturen in der Klimakammer erreichten die Inzuchtarbeiterinnen stets weniger hohe Temperaturen als die Nicht-Inzuchtarbeiterinnen. Dieser Mangel an Regulierfähigkeit bei den Inzuchtvölkern kann dazu führen, daß sie im Frühjahr die Brutaufzucht erst später beginnen als die Nicht-Inzuchtvölker, denn eine konstant hohe Brutnesttemperatur von etwa $35{ }^{\circ} \mathrm{C}$ ist für die heranwachsende Brut unbedingt erforderlich.

Es stellte sich die Frage, ob durch die Kreuzung von ingezüchteten Linien ein Heterosiseffekt erzielt werden kann, der die nachteiligen Auswirkungen der Inzucht auf das Merkmal Temperaturregulierung zum Verschwinden bringt. Heterosis wurde für die verschiedensten Merkmale bei Tieren und Pflanzen nachgewiesen (Nordskog und Ghostley, 1954, Merritt und Gowe, 1960). Bei der Honigbiene wurde Heterosis für einige morphologische Merkmale nachgewiesen (RoBerts, 1961), sowie für die Merkmale Honigertrag und Legeleistung der Königinnen (Cale und Gowen, 1956; Plass, 1953; RutTner, 1968), jedoch noch für keine definierte physiologische Einzelfunktion der Arbeiterinnen.

In den vorliegenden Versuchen wurde das Temperaturregulierungsvermögen von zwei Inzuchtlinien sowie das der Hybriden der beiden Linien getestet. Wie in den vorangegangenen Untersuchungen wurden nur die kältebedingten Reaktionen der Bienen gemessen.

\section{MATERIAL UND METHODE}

Die Versuche wurden mit Arbeiterinnen von Apis mellifica carnica durchgeführt. Es standen 2 Inzuchtlinien zur Verfügung, die nicht verwandt waren, sowie Hybriden der beiden Linien. Alle Kreuzungen waren mit Hilfe der künstlichen Besamung durchgeführt worden. In Abbildung 1 ist das Kreuzungschema wiedergegeben. Der Inzuchtkoeffizient (F), der dem Homozygotiegrad der Tiere entspricht, betrug bei den untersuchten Inzuchtarbeiterinnen $87,5 \%$. Der Inzuchtkoeffizient der Hybriden beträgt $0 \%$. Die Versuche wurden mit Arbeiterinnen von einem Volk der Linie $1\left(I_{1}\right)$, zwei Völkern der Linie $2\left(I_{2 \mathrm{a}}, I_{2 \mathrm{~b}}\right)$ und zwei Völkern der Hybriden $\left(\mathrm{H}_{1}, \mathrm{H}_{2}\right)$ durchgeführt.

Den Völkern wurden Waben mit verdeckelter Brut entnommen und im Brutschrank bei $35{ }^{\circ} \mathrm{C}$ zum Schlüpfen gebracht. Je 50 frisch geschlüpfte Arbeiterinnen eines Volkes wurden in Plexiglaskäfige (MaBe $9 \times 5 \times 6 \mathrm{~cm}$ ) gesetzt und mit frischem Pollen, Honig und Wasser versorgt. Bis zum Alter von 10 Tagen wurden die Bienen bei $30^{\circ} \mathrm{C}$ im Dauerdunkel gehalten und dann in einen Versuchsraum gebracht, in dem gelbes Dauerlicht brannte. Alle Kästchen, zwei pro Volk, wurden in zufälliger Reihenfolge nebeneinander aufgestellt. Die Messungen wurden bei einer Umgebungstemperatur von $20{ }^{\circ} \mathrm{C}$ durchgeführt, es wurde ein Meßgerät benutzt, das einen Stechfühler besaß $(\varnothing 18 \mathrm{~mm})$, der durch die seitlichen Luftlöcher in die 


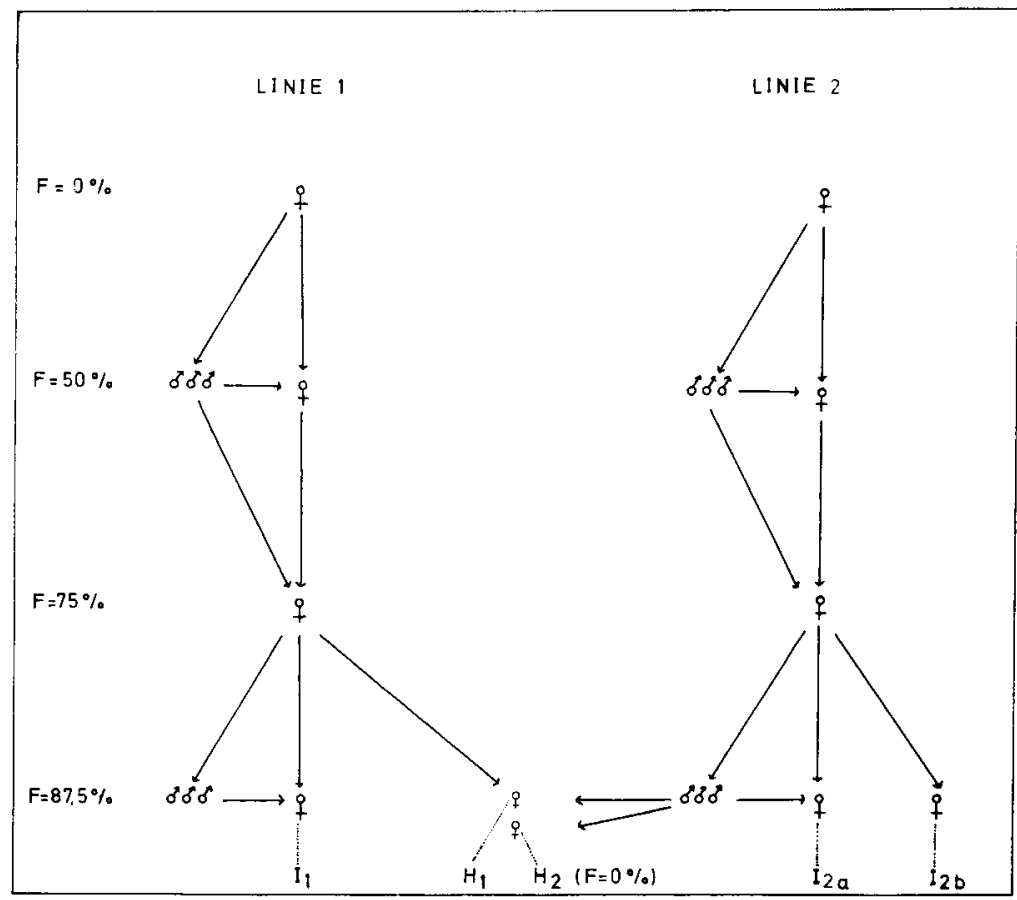

Ав в. 1. - Kreuzungsschema der Linie 1 und 2, sowie der Hybriden aus diesen Linien. Die Kreuzungen wurden alle mit Hilfe der künstlichen Besamung erzielt.

$\mathbf{F}=$ Inzuchtkoeffizient

Fıc. 1. - Schéma de croisement des lignées 1 et 2 et de leurs hybrides. Les croisements ont été obtenus par insémination artificielle

$\mathrm{F}=$ coefficient de consanguinité

Kästchen eingeführt werden konnte. Die Temperatur der wärmsten Stelle in den Kästchen wurde drei Tage lang stündlich von 8-18 h gemessen; die Messungen verursachen keine größeren Störungen unter den Bienen. Für genauere Angaben zur Aufzucht und Versuchsdurchführung siehe BrüCKNER, 1975.

Bei der Auswertung wurden die von den Inzuchtarbeiterinnen der Völker $I_{2 a}$ und $I_{2 b}$ erreichten Temperaturen, sowie die der Hybridvölker $H_{1}$ und $H_{2}$ jeweils gemeimsam behandelt, da sie nicht signifikant verschieden waren. Alle Werte, deren Irrtumswahrscheinlichkeit unter $5 \%$ liegt, wurden als signifikant bezeichnet.

\section{ERGEBNISSE UND DISKUSSION}

Die dreitägigen Messungen ergaben, daß die im Mittel erreichte Temperatur der Hybridarbeiterinnen signifikant über denen der beiden Inzuchtlinien $\operatorname{lag}$ (Linie 1, $\mathrm{p}<0,05 ;$ Linie 2, $\mathrm{p}<0,001$ ). Die Varianzen der von den Hybriden erreichten Temperaturen waren dagegen signifikant kleiner als die der Inzuchtlinien (Linie $1, p<0,01$; Linie $2, \mathrm{p}<0,05$ ). 


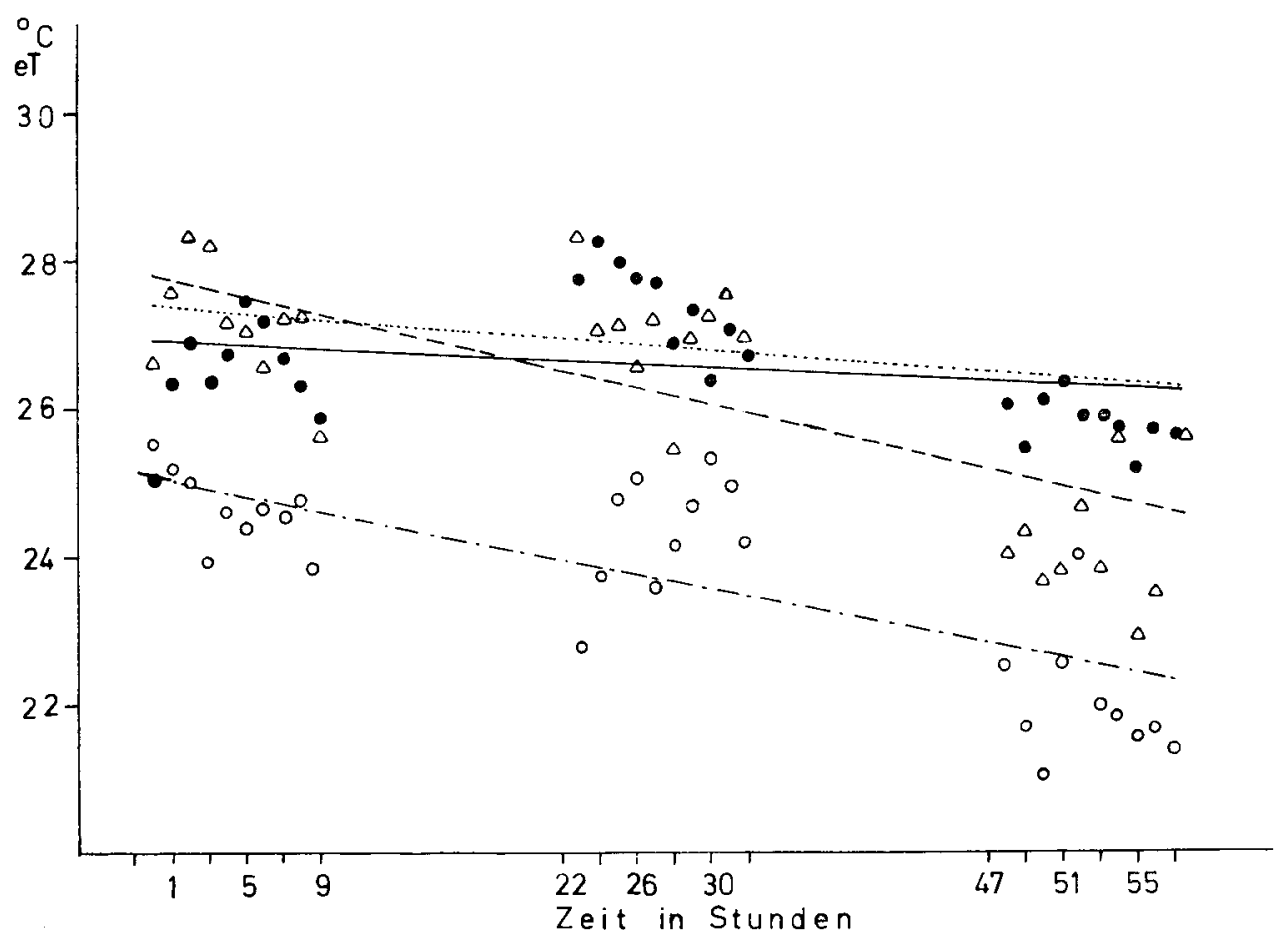

Aвв. 2. - Mittelwerte der erreichten Temperaturen ( $T$ ) der Inzuchtlinien und der Hybriden für die stündlichen Messungen und Regressionsgeraden der erreichten Temperaturen. Zum Vergleich wurde die Regressionsgerade der erreichten Temperaturen von anderen Nicht-Inzuchtarbeiterinnen aus vorangegangenen Versuchen (BRÜCKNER, 1975) punktiert eingezeichnet. Die Umgebungstemperatur während der Versuche betrug $20^{\circ} \mathrm{C}$.

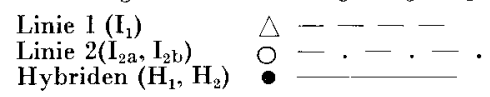

FIG. 2. - Valeurs moyennes atteintes par les lignées consanguines et les hybrides selon les mesures horaires et droites de régression des tenpératures atteintes. A titre de comparaison on a fait figurer en pointillés la droite de régression des températures atteintes par d'autres abeilles non consanguines lors d'expériences antérieures (BRÜCKNER, 1975). La température ambiante durant les expériences atteignait $20^{\circ} \mathrm{C}$.

En abscisses : temps en heures
Lignée $1\left(\mathrm{I}_{1}\right)$
Lignée $2\left(\mathrm{I}_{2}, \mathrm{I}_{2}\right)$
Hybrides $\left(\mathrm{H}_{1}, \mathrm{H}_{2}\right)$

In Abbildung 2 sind die Mittelwerte der bei den stündlichen Messungen von Inzucht- und Hybridarbeiterinnen erreichten Temperaturen wiedergegeben, sowie die Regressionsgeraden der erreichten Temperaturen.

Die Regressionsgeraden der Inzuchtlinien sind signifikant von der der Hybriden verschieden, beide weisen einen starken Abfall auf, während der Abfall der Regressionsgeraden der Hybriden nicht signifikant von null ver- 
schieden ist. Untereinander sind die Regressionsgeraden der Inzuchtarbeiterinnen nicht signifikant verschieden. Bei einem Vergleich der von den Hybriden erreichten Temperaturen mit den von Nicht-Inzuchtarbeiterinnen in vorangegangenen Versuchen erreichten Temperaturen (BRǗcKNER, 1975) zeigte sich, daß die Temperaturen der Hybriden denen der Nicht-Inzuchtarbeiterinnen entsprechen (Abb. 2).

Aus den vorliegenden Ergebnissen wird deutlich, daß ein Heterosiseffekt für das Merkmal Temperaturregulierung bei den Hybriden auftritt. Die von den Hybriden im Mittel erreichten Temperaturen liegen über denen der Inzuchtlinien und weisen zudem geringere Schwankungen auf als bei letzteren. Ein Temperaturabfall der erreichten Temperaturen mit der Zeit ist bei den Hybriden kaum vorhanden, während er bei beiden Inzuchtlinien stark ausgeprägt auftritt.

Wie gezeigt werden konnte, ist die verminderte Fähigkeit zur Temperaturregulierung bei ingezüchteten Arbeiterinnen an ihren Mangel an genetischer Variabilität gebunden (BR ÜCKNER, 1975). Das Homozygotwerden zahlreicher Loci im Genom führt offensichtlich zu einem Verlust an Pufferungskapazität. In weiteren Versuchen (BRÜCKNER, im Druck) konnte gezeigt werden, daß die schlechtere Temperaturregulierung der Inzuchtbienen, zumindest zum Teil, durch eine verminderte Abgabe von Stoffwechselwärme bedingt ist.

Die wiedergewonnene Heterozygotie der Hybridarbeiterinnen $(F=0 \%)$ behebt diesen Mangel und führt zu einer Leistungsfähigkeit, die der von NichtInzuchtvölkern entspricht.

In der vorliegenden Arbeit wurden nur Hybridarbeiterinnen des einen Kreuzungsmodus getestet (Königinnen der einen Linie gekreuzt mit Drohnen der anderen Linie). Da die Heterozygotie der Arbeiterinnen von Bedeutung ist und der Inzuchtkoeffizient der Hybriden bei Kreuzung von nicht-verwandten Inzuchtlinien stets $0 \%$ beträgt, muß jedoch angenommen werden, daß die reziproke Kreuzung zum selben Ergebnis bezüglich der Temperaturregulierung führen würde.

Für die Praxis der Bienenzucht ist es von Bedeutung, daß die negativen Auswirkungen der Inzucht auf das Merkmal Temperaturregulierung bei Kreuzung von nicht verwandten Inzuchtlinien durch den bei den Hybriden auftretenden Heterosiseffekt wieder aufgehoben werden können.

$$
\begin{array}{r}
\text { Eingegangen im März } 1976 . \\
\text { Reģu pour publication en mars } 1976 .
\end{array}
$$

\section{DANK}

Herrn Professor Dr. F. Rutrner möchte ich sehr herzlich für die Ermöglichung dieser Untersuchungen im Institut für Bienenkunde in Oberursel danken, sowie Herrn Dr. V. MAUl für die Überlassung des Bienenmaterials. 


\section{RÉSUMÉ}

On a testé la faculté de régulation thermique sur des petits groupes d'ouvrières de 2 lignées fortement consanguines (coefficient de consanguinité $87,5 \%$ ) d'Apis mellifica carnica et sur leurs hybrides. On a fait éclore à $35{ }^{\circ} \mathrm{C}$ du couvain operculé et placé les ouvrières, à raison de 50 par colonie, dans des cages en plexiglas. On les a nourries avec du pollen frais, de l'eau et du miel et conservées à l'obscurité et à $30^{\circ} \mathrm{C}$ pendant les 10 premiers jours de leur vie. Les mesures ont été effectuées à la température ambiante de $20^{\circ} \mathrm{C}$. Durant 3 jours on a mesuré toutes les heures de 8 à $18 \mathrm{~h}$ les températures atteintes par les abeilles dans les cagettes à l'aide d'un thermistor.

Les valeurs des températures moyennes atteintes par les hybrides sont significativement plus hautes que celles atteintes par les deux lignées consanguines et présentent des fluctuations significativement plus faibles. Chez les hybrides c'est à peine s'il y a une chute en fonction du temps des températures atteintes, alors que cette chute est fortement marquée chez les deux lignées consanguines. Il n'existe pas de différences significatives entre les températures atteintes par les hybrides et celles atteintes par des ouvrières non consanguines.

Les résultats prouvent l'existence du phénomène d'hétérosis pour le caractère régulation thermique. L'amélioration de la régulation thermique chez les hybrides doit provenir de l'hétérozygotie retrouvée des ouvrières. En croisant deux lignées consanguines non parentes il est par conséquent possible de redévelopper la faculté des ouvrières à réguler leur température.

\section{LITERATURVERZEICHNIS}

BrüGKner D., 1975. - Die Abhängigkeit der Temperaturregulierung von der genetischen Variabilität der Honigbiene (Apis mellifica). Apidologie 6 (4), 361-380.

BRÜCKNER D., 1976. - Vergleichende Untersuchungen zur Temperaturpraeferenz von ingezüchteten und nicht-ingezüchteten Arbeiterinnen der Honigbiene (Apis mellifica). Apidologie (im Druck).

Cale G.H. and J.W. Gowen, 1956. - Heterosis in the honeybee (Apis mellifera L.). Genetics 41, 292-301.

Merritt E.S. and R.S. Growe, 1960. - Combining ability among breeds and strains of meat type fowl. Canad. J. genet. Cytol., 3, 286.

Nordskog A.W. and F.J. Ghostley, 1954. - Heterosis in poultry. Poultry Sci. 33704.

Plass F., 1953. - Inzuchtwirkung und Heterosiseffekt bei der Honigbiene. Schriftreihe der $A I D$, H. 66 Bonn.

Roberts W.C., 1961. - Heterosis in the honeybee as shown by morphological characters in inbred and hybrid bees. Ann. Ent. Soc. Am. 54, 878-882.

RutTner F., 1968. - Methods of breeding honeybees : Intra-racial selection of inter-racial hybrids. Bee World 49. 66-72. 